\title{
HEARING THE SHAPE OF AN ANNULAR DRUM
}

\author{
H. P. W. GOTTLIEB'
}

(Received 16 October 1981; revised 19 July 1982)

\begin{abstract}
The asymptotic expansion for a spectral function of the Laplacian operator, involving geometrical properties of the domain, is demonstrated by direct calculation for the case of a doubly-connected region in the form of a narrow annular membrane. By utilizing a known formula for the zeros of the eigenvalue equation containing Bessel functions, the area, total perimeter and connectivity are all extracted explicitly.
\end{abstract}

\section{Introduction}

During the past couple of decades, techniques have been developed for obtaining information on geometrical properties of a closed manifold, such as its volume, from asymptotic expansions of spectral functions involving eigenvalues of Laplacian-type operators. (See [2], [4], [6], [7] and [8].) Kac's paper [6], is particularly stimulating: the title of this paper and its contents may be regarded as being in response to the question posed in the title of Kac's paper. As well as dealing with the case of a vibrating membrane, Kac also considers several analogous physical systems from the fields of classical and quantum statistical mechanics, diffusion theory and Brownian motion.

The result which concerns us here, in the form discussed by Kac [6], is as follows. Consider the eigenvalues $\lambda_{n}$ of the Helmholtz equation (obtained from the two-dimensional wave equation by separation of variables after taking out the time dependence):

$$
\frac{1}{2} \nabla^{2} \psi_{n}+\lambda_{n} \psi_{n}=0
$$

\footnotetext{
${ }^{1}$ School of Science, Griffith University, Nathan, Queensland 4111.

(1) Copyright Australian Mathematical Society 1983
} 
For a vibrating membrane held fixed along its smooth boundaries, with $r$ smooth holes, there is the asymptotic relation

$$
\sum_{n=1}^{\infty} \exp \left(-\lambda_{n} t\right) \sim \frac{A}{2 \pi t}-\frac{L}{4} \frac{1}{\sqrt{2 \pi t}}+\frac{(1-r)}{6} \text { as } t \rightarrow 0,
$$

where $A$ is the area of the membrane and $L$ is the total length of its perimeter.

It is the purpose of this note to illustrate the theorem by direct calculation for the case of a multiply-connected region, something which does not appear to have been done before. We use the case of a vibrating annular membrane with fixed rims, and extract explicitly its area, perimeter and connectivity.

$\mathrm{Kac}$ [6], as well as other more rigorous analyses (e.g. [2]), proceeded indirectly by consideration of the Green's function of a corresponding diffusion equation. But even for the circular membrane, Kac was unable to proceed further than the first two terms on the right-hand side of (2) because of difficulty with the Green's function. For a polygonal drum, letting the number of sides become infinite, Kac obtained the constant term $\frac{1}{6}$ corresponding, for $r=0$, to the third term in (2). Subsequently, McKean and Singer [7] proved (2) from a differential geometric approach on a more general manifold.

Here, we are able to proceed directly by using a known expression for the eigenvalues on the left-hand side of equation (2) for the case of a narrow annular membrane. The harmonic properties of such an annular drum have been investigated by the author [5]. For a full circular membrane, there is an asymptotic expression for the $s$ th zero of the corresponding eigenvalue equation, valid for large $s$ (Abramowitz and Stegun [1], page 371). However, the infinite sum in equation (2) must account for all eigenvalues, so such an asymptotic expression does not permit the accurate inclusion of all summands. For an annulus, the corresponding expression ([1], page 374) may be used for all $s$ provided the ratio of external to internal radii is sufficiently close to 1, [3].

\section{Derivation}

For the wave equation of a system vibrating with angular frequency $\omega$, the standardized parameter in equation (1) is given by $\lambda_{n}=k^{2} / 2$ where $k=\omega / c$ and $c$ is the free wave speed. In the case of the annular membrane [5], $k=x_{N}^{(s)} / a$, where $x_{N}^{(s)}$ is the $s$ th root of the "cross-product" Bessel equation

$$
J_{N}(\gamma x) Y_{N}(x)-J_{N}(x) Y_{N}(\gamma x)=0,
$$

where $J_{N}$ and $Y_{N}$ are the Bessel functions of integer order $N$ of the first and second kind respectively, and the radius ratio is $\gamma=b / a>1$. Here, $s$ is the radial number and $N$ is the angular number. 
McMahon's formula ([1], page 374) for $x_{N}^{(s)}$ may be written in the form

$$
x_{N}^{(s)}=\frac{\pi s}{\gamma-1}+\frac{4 N^{2}-1}{8 \pi \gamma}\left(\frac{\gamma-1}{s}\right)+O\left[\left(\frac{\gamma-1}{s}\right)^{3}\right] .
$$

Note that the parameter $(\gamma-1) / s$ is small not only if $s$ is large but also if $\gamma-1$ is small, i.e. for a narrow annulus. Thus we have

$$
\lambda_{N}^{(s)}=\frac{1}{2 a^{2}}\left[\frac{\pi^{2} s^{2}}{(\gamma-1)^{2}}+\frac{N^{2}}{\gamma}-\frac{1}{4 \gamma}+O(\gamma-1)^{2}\right],
$$

and we wish to evaluate the expression

$$
E=\sum_{s=1}^{\infty} \sum_{N=-\infty}^{\infty} \exp \left(-\lambda_{N}^{(s)} t\right)
$$

as $t \rightarrow 0$ in the case of a narrow annulus, for which $\gamma$ is sufficiently close to 1 , so the last term in equation (5) may be neglected. In (6), the summation takes account of the double degeneracy for angular modes with $N>0$.

To evaluate the sum (6) we utilize the Poisson summation formula ([10], page 219) which is related to the transformation formula for the Jacobi theta function ([9], page 74) and may conveniently be written in the form

$$
\begin{aligned}
\sum_{n=-\infty}^{\infty} \exp \left(-z n^{2}\right) & =(\sqrt{\pi} / \sqrt{z}) \sum_{n=-\infty}^{\infty} \exp \left(-\pi^{2} n^{2} / z\right) \\
& \sim \sqrt{\pi} / \sqrt{z} \quad \text { as } z \rightarrow 0 ;
\end{aligned}
$$

and

$$
\sum_{n=1}^{\infty} \exp \left(-z n^{2}\right) \sim \sqrt{\pi} /(2 \sqrt{z})-\frac{1}{2} \quad \text { as } z \rightarrow 0 .
$$

The corrections to the asymptotic expressions (7b) and (7c) are exponentially small errors ([7], page 67), and do not affect the calculations involving powers of $\sqrt{t}$. Factorizing the exponentials in (6) resulting from use of (5), and evaluating the sums accordingly to (7b) and (7c) as $t \rightarrow 0$, we find

$$
\left.\begin{array}{rl}
E & \sim A / 2 \pi t+O\left[(\gamma-1)^{3} / t\right] \\
& -L / 4 \sqrt{2 \pi t}+O\left[(\gamma-1)^{2} / \sqrt{t}\right] \\
& +0+O(\gamma-1) \\
& -\sqrt{2 \pi t} / 16 a+O[(\gamma-1) \sqrt{t}] \\
& +O[(\gamma-1) t],
\end{array}\right\}
$$

where $A=\pi a^{2}\left(\gamma^{2}-1\right)=\pi\left(b^{2}-a^{2}\right)$ and $L=2 \pi a(\gamma+1)=2 \pi(b+a)$. 
Insofar as the first three sets of terms in (8) involve respectively an area, a length, and a pure number independent of the dimensions of the system, and as the corrections to each of the first three successive terms in (8) decrease by a power of $(\gamma-1)$ for each increase of $\frac{1}{2}$ in the power of $t$, we have verified the formula (2) for our case of a narrow $(\gamma-1$ small) vibrating annulus $(r=1)$. Of special interest is the recovery of the term involving the total length $L$ which is not small. The area, perimeter and connectivity of the annulus have all heen evinced because of the analytical form of McMahon's formula (4) which involves the two independent summation parameters $s$ and $N$ in that particular combination resulting from the solution of the appropriate eigenvalue equation (3). A concrete case of the asymptotic relation (2) has thus been demonstrated explicitly for a multiply-connected region.

The next term in (8), involving $\sqrt{2 \pi t}$, whose coefficient has dimensions of inverse length, is included to allow comparison with any future attempts to deduce its geometrical interpretation in a general formulation.

\section{Acknowledgement}

The author wishes to thank Dr. A. J. Bracken and Dr. A. J. O'Connor for drawing attention to several references.

\section{References}

[1] M. Abramowitz and I. A. Stegun (eds.), Handbook of mathemattcal functions (Dover, New York, 1972).

[2] R. Balian and C. Bloch, "Distribution of eigenfrequencies for the wave equation in a finite domain I", Ann. Physics 60 (1970), 401-447.

[3] S. De, "Vibrations of a non-homogeneous annular membrane", J. Indian Math. Soc. 36 (1972), 297-305.

[4] M. E. Fisher, " On hearing the shape of a drum", J. Combin. Theory 1 (1966), 105-125.

[5] H. P. W. Gottlieb, "Harmonic properties of the annular membrane", J. Acoust. Soc. Amer. 66 (1979), 647-650.

[6] M. Kac, "Can one hear the shape of a drum?", Amer. Math. Monthly 73 (1966), 1-23.

[7] H P. McKean and I. M. Singer, "Curvature and the eigenvalues of the Laplacian", $J$. Differential Geom. 1 (1967), 43-69.

[8] M. Reed and B. Simon, Methods of modern mathematical physics, Vol. IV (Academic, 1978), Chapter 13.

[9] A. Sommerfeld, Partial differential equations in physics (Academic, 1964).

[10] R. M. Ziff, G. E. Uhlenbeck and M. Kac, "The ideal Bose-Einstein gas, revisited”, Phys. Rep. 32 (1977), 169-248. 